\title{
04 | La Arquitectura como construcción de lo SOCIAL en los dibujos de Alison y Peter Smithson: De la calle como figuración de lo relacional a la desaparición de la escala y los límites Architecture as construction of the SOCIAL in Alison and Peter Smithson's drawings: From the street as figu- ration of the relational to the disappearance of the scale and boundaries_ Iván Capdevila Castellanos
}

La figura 1 [1] muestra el último de una serie de dibujos realizados por Alison y Peter Smithson entre 1951 y 1954 en relación a su propuesta de concurso para Golden Lane en 1952. En primer lugar, este dibujo destaca porque no manifiesta su escala o límites ni su contexto físico. Estos aspectos lo convierten en un dibujo único, especialmente si entendemos que se realiza en el contexto de los CIAM. Una de las principales modificaciones que introdujo la nueva generación de arquitectos en los CIAM IX y CIAM X, liderados por Alison y Peter Smithson, entre otros, fue la de debatir la arquitectura sobre la base de proyectos reales; es decir, proyectos que perteneciesen a la práctica profesional de sus participantes. Su título es Diagram of Appreciated Unit. Peter Smithson define "Appreciated Unit" así:

"Una unidad apreciada no es un "grupo visual" o un "vecindario", sino una parte de una aglomeración humana que puede ser "sentida" $1 "$.

La aglomeración humana a la que Peter Smithson se está refiriendo puede ser cualquier entorno sujeto a la experiencia: un edificio, una calle, un barrio o una ciudad. El dibujo, al no tener escala ni límites y al no hacer referencia a un contexto físico específico permite, precisamente, esta ambigüedad articulada por su título.

Para entender la contemporaneidad de este dibujo y la relevancia ideológica en la posición de los Smithson es necesario conocer la secuencia de dibujos que construyen sus antecedentes.

\section{Patrick Geddes como herramienta estratégica de transición en la política de los} Smithson

\section{- La manipulación del concepto de Hábitat}

Los dos dibujos que componen el diagrama de la figura 2 [2] vienen a referirse a una misma cosa: las distintas escalas de asociación humanas. Estas son la gran ciudad, la ciudad, el pueblo y la vivienda dispersa. Del mismo modo, en el contexto de un valle y un río que lo cruza, podemos entender que la gran ciudad adopta una posición central, vinculada al río, mientras el resto de asociaciones se producen de manera simétrica en las distintas alturas del valle. Además, el dibujo inferior nos muestra las distintas densidades urbanas de las distintas aglomeraciones.
Resumen pág 65 | Bibliografía pág 69

Universidad de Alicante. Iván Capdevila Castellanos, Doctor Arquitecto por la Universidad de Alicante y Máster en Arquitectura por la Bartlett School en Londres (U.C.L.). Combina su actividad docente e investigadora como profesor de Proyectos Arquitectónicos en la UA con su práctica en PLAYstudio, que ha sido premiada en numerosos concursos internacionales, destacando cuatro primeros premios en Europan. capdevila@ua.es

\section{Palabras clave}

Social, arquitectura relacional, arquitectura inclusiva, Smithsons, calle, dibujo, cotidianidad, ecología, arbitrariedad Social, Relational Architecture, Inclusive Architecture, Smithsons, street, drawing, ordinariness, ecology, randomness

Método de financiación Financiación propia

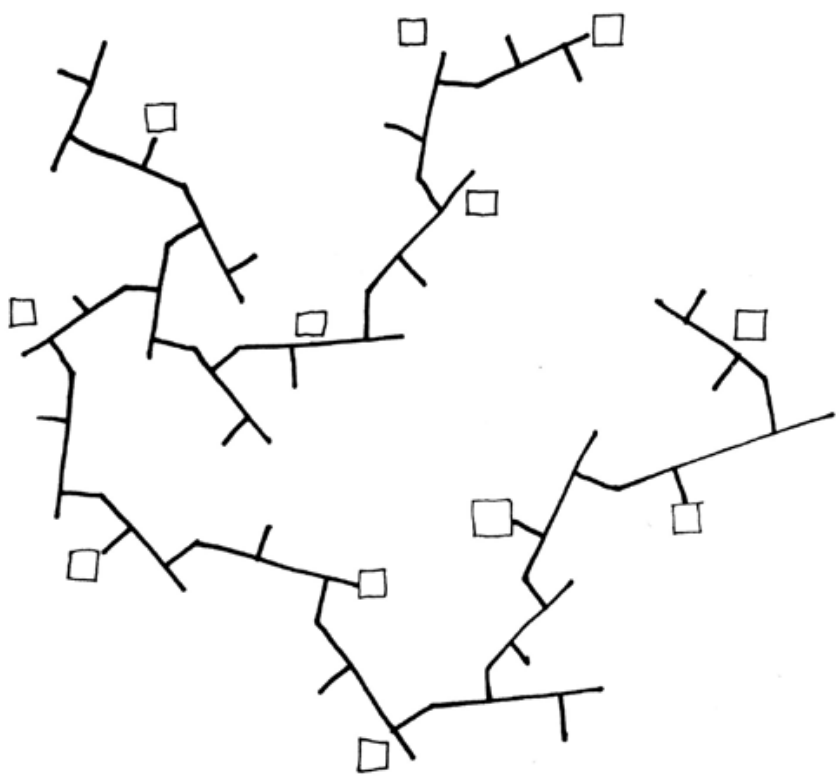


1 SMITHSON, Alison; SMITHSON, Peter. Urban structuring: studies of Alison \& Peter Smithson. New York: Studio Vista, 1967, p. 20.

2 Artículo escrito en 1949 y recogido en: GIEDION, Sigfried. Space, time, and architecture; the growth of a new tradition. Cambridge: Harvard University Press, 1956.

${ }^{3}$ La revista Architectural Review se haría eco de este debate entre 1948 y 1950.

${ }^{4}$ El tema de la humanización de la ciudad sería un tema también tratado anteriormente por Sigfried Giedion en 1949 y recogido en su libro de 1956.

5 Los proyectos presentados tenían en común un deseo de crear entornos que fomentaran relaciones entre sus habitantes, entre un edificio y su entorno, y que albergasen las necesidades culturales de la gente. Mientras los Smithson encontraron la inspiración en las fotos de Nigel Henderson, GAMMA tuvo como punto de partida la vida cotidiana y edificación en el "bidonville". Su análisis estaba enraizado en la larga tradición de investigación etnológica de las colonias francesas. Sin embargo, a diferencia de los Smithson, su mirada era distante, casi antropológica, o desde la sorpresa o curiosidad generada por el que descubre algo nuevo, exótico, si cabe.
The Valley Section fue el diagrama que los Smithson utilizaron para ilustrar el documento preparatorio para el CIAM X (1956). En este, la palabra "Hábitat" articula su contenido. Bajo propuesta de Le Corbusier, el tema para el CIAM IX sería el "Hábitat". Este término proveniente de la ecología, por entonces una ciencia no muy mayor y poco familiar, hace referencia no tanto al análisis de organismos separados y funciones sino más bien a sus relaciones mutuas y la interacción con el entorno. Sería, de hecho, Sigfried Giedion quien, remitiéndose al Dr. Scott Williamson ${ }^{2}$, utilizaría el concepto de "hábitat" en el mismo sentido que lo hacía la ecología. Sin embargo, la noción de hábitat de Le Corbusier era en realidad muy limitada. No era más que la de la forma construida de la familia ${ }^{3}$. Y así, la Charte de l'Habitat -Carta del Hábitat- se convertiría pronto en una carta de la vivienda como continuación de la Carta de Atenas. Fue, precisamente, en el CIAM IX donde Alison y Peter Smithson conocerían a Jaap Bakema, Aldo Van Eyck y George Candilis. Este último sería uno de los más críticos con el concepto de "Hábitat" de Le Corbusier y lideraría una reunión informal en Sigtuna previa al CIAM IX de 1953, en la que se plantea la cuestión del "hábitat" como fundamental para redefinir el urbanismo. Sin embargo, la noción de "hábitat" de este grupo de jóvenes arquitectos no sería la de una aproximación organicista al planeamiento sino más bien "humana" ${ }^{4} \mathrm{o}$, mejor, entendida desde sus relaciones sociales ${ }^{5}$.

Así pues, Alison y Peter Smithson ya en 1951 se vieron enfrentados a la cuestión del "Hábitat" como concepto fundamental para el desarrollo de nuestras ciudades. Años más tarde en su artículo "The Built World: Urban Reidentification" publicado en Architectural Design (junio de 1955) contextualizaban su propuesta para el concurso de Golden Lane (1952) dentro de "una aproximación ecológica al problema del hábitat."

Por otro lado, en el primer borrador para el Manifiesto de Doorn, en 1954, Peter Smithson vincula la palabra "Hábitat" al dibujo. Es aquí donde encontramos el primer dato fundamental ya que se refiere a su dibujo como "Geddes valley section" -la sección del valle de Geddes- en referencia al biólogo, sociólogo y urbanista escocés Patrick Geddes (1854-1932). Si observamos en detalle su Valley Section [3], entenderemos que este diagrama establece vínculos fuertes, tanto conceptuales como históricos, entre estructuras sociales y la forma construida de asentamientos urbanos. La relación entre estructura social y su forma construida es algo en lo que los Smithson trabajarían insistentemente.

La "Sección del Valle" [3] es una sección longitudinal que empieza arriba en las montañas y, bajando por el curso de un río, atraviesa una llanura para desembocar en la costa. Y esta sección no incluye un solo valle sino varios. Dentro de esta región, Geddes está inscribiendo varios significados. En la parte inferior, relaciona las distintas localizaciones geográficas con sus oficios "naturales". Como el propio Geddes decía: "si estos oficios convivían en harmonía con su entorno particular, las sociedades deberian materializarse bajo la forma de tales asentamientos humanos tal y como pueden verse en la sección del valle". Para Patrick Geddes, toda la región representada en la sección del valle es una sola entidad, una sola ciudad.

Puede ser que esta sea la principal similitud entre la sección del valle de Geddes y la interpretación de los Smithson. De hecho, lo que está haciendo Geddes es considerar los distintos tipos de asentamientos en la región del valle como una expresión de una interacción armónica

[2]

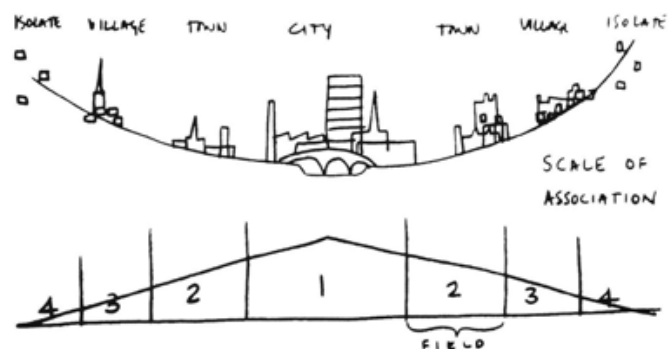

[3]

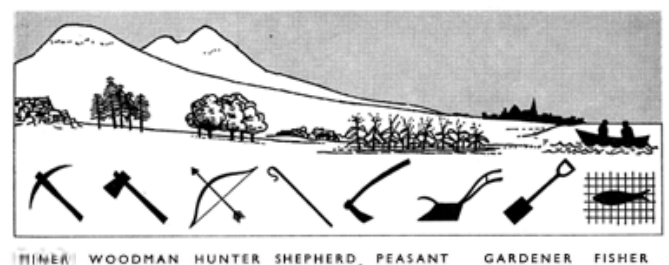

[1] Alison y Peter Smithson, Diagram of Appreciated Unit (Diagrama de una Unidad Apreciable), 1954. Fuente: ROUILLARD, Dominique. Superarchitecture: le futur de l'architecture, 1950-1970. Paris: Editions de la Villette, 2004, p. 30

[2] Peter Smithson, The Valley Section Diagram (Diagrama de la Sección del Valle), 1951. Fuente: SMITHSON, Alison. SMITHSON, Peter. The charged void, Urbanism. New York: Monacelli Press, 2004, p. 24 


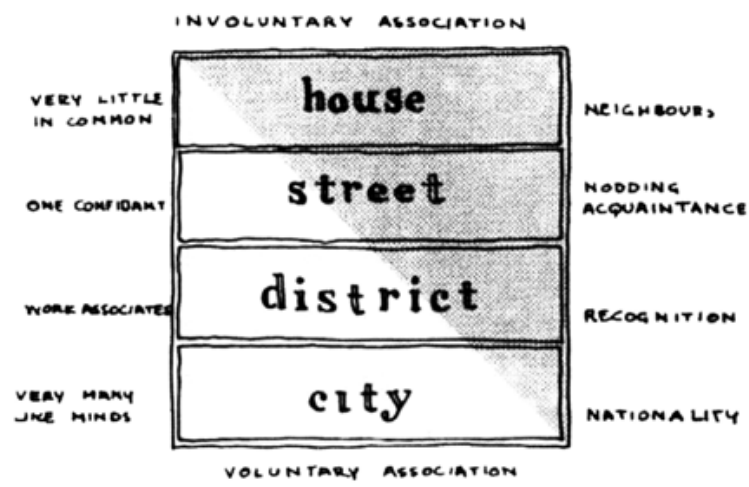

[4] ) Alison y Peter Smithson, Scale of Association (Escala de Asociación), 1951. Fuente: SMITHSON, Alison; SMITHSON, Peter. Urban structuring: studies of Alison \& Peter Smithson. New York: Studio Vista, 1967, p. 20.

[4]

e histórica de las distintas ocupaciones "naturales" del valle con sus respectivos entornos. Pero esta lectura es más bien histórica: los Smithson la evolucionan hacia una lectura más contemporánea, en la que el principal énfasis es el de proponer una arquitectura que refleje y responda de una manera particular a su entorno.

Además, el dibujo de los Smithson [2] es intencionadamente simétrico -conceptualmente-, encontrándose en el centro del mismo la gran ciudad. De hecho, todas las aglomeraciones representadas son urbanas $\mathrm{y}$, desde luego, nada tienen que ver con la relación que los distintos oficios rurales, artesanales, etc., establecen con determinados lugares del territorio. $Y$, lo que es más importante, sustituyen la idea de asentamiento por la de asociación. Si bien Geddes siempre relacionaría la idea de comunidad a asentamiento, los Smithson entenderían la comunidad como una jerarquía de elementos de asociación:

"una comunidad debería construirse a partir de una jerarquía de elementos de asociación" 6.

Justamente este interés por las relaciones de asociación en el hecho construido será la principal aportación de los Smithson sobre el concepto de Hábitat para el CIAM X.

\section{- La manipulación del 4}

"Este estudio (...) intenta expresar los distintos niveles de asociación -la casa, la calle, el distrito, la ciudad." 7

Uno de los rasgos principales del dibujo mostrado en la figura 4 [4] es la clasificación taxativa que hace de cuatro entornos humanos: la casa, la calle, el distrito y la ciudad. Cada uno de ellos representa, a su vez, un nivel de "asociación" diferenciado.

Dos años más tarde, para el CIAM IX [5], los Smithson presentan Urban Re-Identification: una matriz de dibujos suyos y fotografías realizadas por Nigel Henderson en el barrio de Bethnal Green en Londres. Los dibujos de propuesta se agrupan, precisamente, siguiendo las cuatro categorías del diagrama de la figura 4. [4]

La ciudad que nos plantean los Smithson se puede entender desde cuatro escalas distintas: la casa, la calle, el distrito y la ciudad. Y cada una de ellas define un entorno urbano cuya identidad se construye según el tipo de asociaciones que se producen. Las palabras "identidad" y "asociación" son, pues, las más repetidas. De hecho, en su texto Urban Structuring reconocen que sus intereses principales en estos momentos giran en torno a los conceptos de identidad y asociación:

"Este estudio se centra en el problema de la identidad en una sociedad en continuo movimiento. Propone que una comunidad debería construirse a partir de una jerarquía de elementos asociativos e intentando expresar los distintos niveles de asociación" ${ }^{8}$.

\section{- La "calle" como figuración de lo real: de la monumentalidad de la Piazza a la cotidi- aneidad de la calle}

The City [6] sería el primer dibujo que daría forma al diagrama Scale of Association [4]. Si prestamos atención a su título, "La Ciudad", pronto entenderemos que este dibujo nos está mostrando simultáneamente las cuatro escalas que se producen en el entorno humano de los Smithson: casa, calle, distrito y ciudad. Es importante señalar cómo en esta ciudad el soporte real se muestra a través de sus elementos más representativos. En ningún caso se reconoce trama urbana alguna. El dibujo The City se presentó en la matriz Urban Re-Identification [5] de 
${ }^{6}$ SMITHSON, Alison; SMITHSON, Peter. Urban structuring: studies of Alison \& Peter Smithson. New York: Studio Vista, 1967,

p. 21.

${ }^{7}$ lbid.

8 Ibid.

${ }^{9}$ En 1959 el mismo Louis Kahn hará suya la definición que los Smithson hacen de la palabra "calle", la de "lugar": "que es una palabra mejor que lo que nunca hubiese podido concebir para definir la calle". Citado en: NEWMAN, Oscar. CIAM '59 in Otterlo. Stuttgart: K. Krämer, 1961, p. 208.

${ }^{10}$ Memoria de concurso para Golden Lane, 1952.

${ }^{11}$ Sobre la calle de los Smithson, en su imagen de una calle, en su retícula para el CIAM IX de 1953.

12 SMITHSON, Alison; SMITHSON, Peter The charged void, Urbanism. New York: Monacelli Press, 2004, p. 24.

13 Judith Henderson y la organización Mass Observation o Peter Wilmott y Michael Young autores del influyente libro de 1957 Family and Kinship in the East End.

14 Peter Smithson, "Prefacio," en WALSH, Victoria. Nigel Henderson: parallel of life and art. London: Thames \& Hudson, 2001, p. 150. los Smithson para el CIAM IX junto a los dibujos del concurso de Golden Lane. Si lo comparamos con la planta de su propuesta de concurso [7], la relación entre ambos dibujos resulta evidente. No obstante, Peter Smithson realizaría otra serie de dibujos [8] [9] que muestran la transición del edificio a la ciudad: La descripción de uno de ellos hecha por éste nos acaba revelando algo que ya parecía obvio: están dibujando una red de "calles-en-el-aire". Pues, si esa red de calles, al conectarse, forma una calle continua y diversa en su recorrido, podremos decir que en Golden Lane es Casa y Calle, en este dibujo es Distrito y, en The City, se convierte, por fin, en Ciudad.

Así pues, The City [6] nos muestra una ciudad que se construye a partir de una red relacional que toma forma de calle continua y que construye, en su recorrido, las cuatro escalas urbanas del entorno humano. Es, en este punto, cuando podemos afirmar que la calle es la diagonal construida y figurada del dibujo Scale of Association [4] o, incluso, la propia línea de Sección del Valle [3] que incluye a los cuatro tipos de comunidad-asociación en una misma región.

"Cada parte de cada "calle-en-el-aire" tendrá suficiente gente accediendo desde ella para llegar a convertirse en una entidad social. Las calles serán lugares ${ }^{9}$ y no pasillos o balcones" ${ }^{10}$.

No podemos obviar que gran parte del amor que los Smithson profesan a la calle se lo deben a los paseos guiados por su gran amigo Nigel Henderson en unas calles de Bethnal Green devastadas por la guerra, en busca de momentos de felicidad colectiva y urbana:

"En los suburbios y barrios marginales la relación vital entre la casa y la calle sobrevive, los niños corren por ahí, la gente se para y habla, los vehículos están aparcados: en los jardines traseros hay palomas y mascotas y las tiendas están a la vuelta de la esquina: conoces al lechero, tú estás fuera de tu casa en tu calle" ${ }^{11 .}$

De hecho, para los Smithson la calle se convertiria en el lugar de expresión individual y colectiva:

"En una sociedad unida viviendo en un desarrollo urbano unido como el de estas calles hay un sentimiento intrínseco de seguridad y vínculo social que tiene mucho que ver con la obviedad y el simple orden de la forma de la calle: alrededor de cuarenta casas enfrentadas a un espacio abierto común... la calle. La calle no es solo un medio de acceso sino también un foro para la expresión social. En las calles, encontramos una relación muy simple entre casa y calle. ¿Cuántos de los actos tradicionales de expresión -de felicidad, pasar el tiempo, fe, jugar, aprender- probablemente quieren seguir encontrando su lugar?

El hogar -entendido como lugar del fuego- y el umbral son símbolos que, conjuntamente, representan para la mayoría la imagen de casa.

Cuarenta o cincuenta casas hacen una buena calle.

Las calles, con muchos equipamientos locales grandes y pequeños en los intersticios y rotondas construyen un distrito claramente reconocible. Los Distritos, intercalados con muchos otros equipamientos, más complejos que lo que ellos mismos podrían soportar, construyen una ciudad. La Casa, la Calle, el Distrito son elementos de La Ciudad" ${ }^{12}$.

Así pues, la calle es un elemento intermedio entre la casa y el distrito que dota de identidad tanto a los unos como a los otros y que construye, a su vez, toda la ciudad. La calle es el símbolo de la expresión personal individual y colectiva, idea a la que la importancia creciente de la sociología contribuye: son cientos los estudios sociológicos que se están llevando en esos momentos a cabo y, especialmente, en Bethnal Green ${ }^{13}$.

"La sociología había comenzado a emerger desde la selva tropical a las calles. Pero tenía que hacerse visible a los arquitectos para que fuesen conscientes de esto como un surgimiento general, y esto es lo que ocurrió para mí y Alison en el CIAM IX en Aix-en-Provence en 1953" 14.

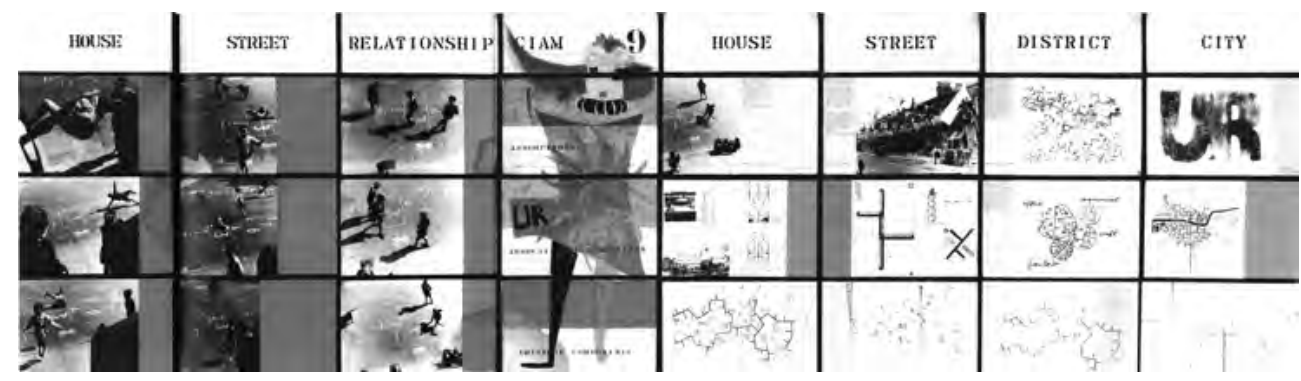




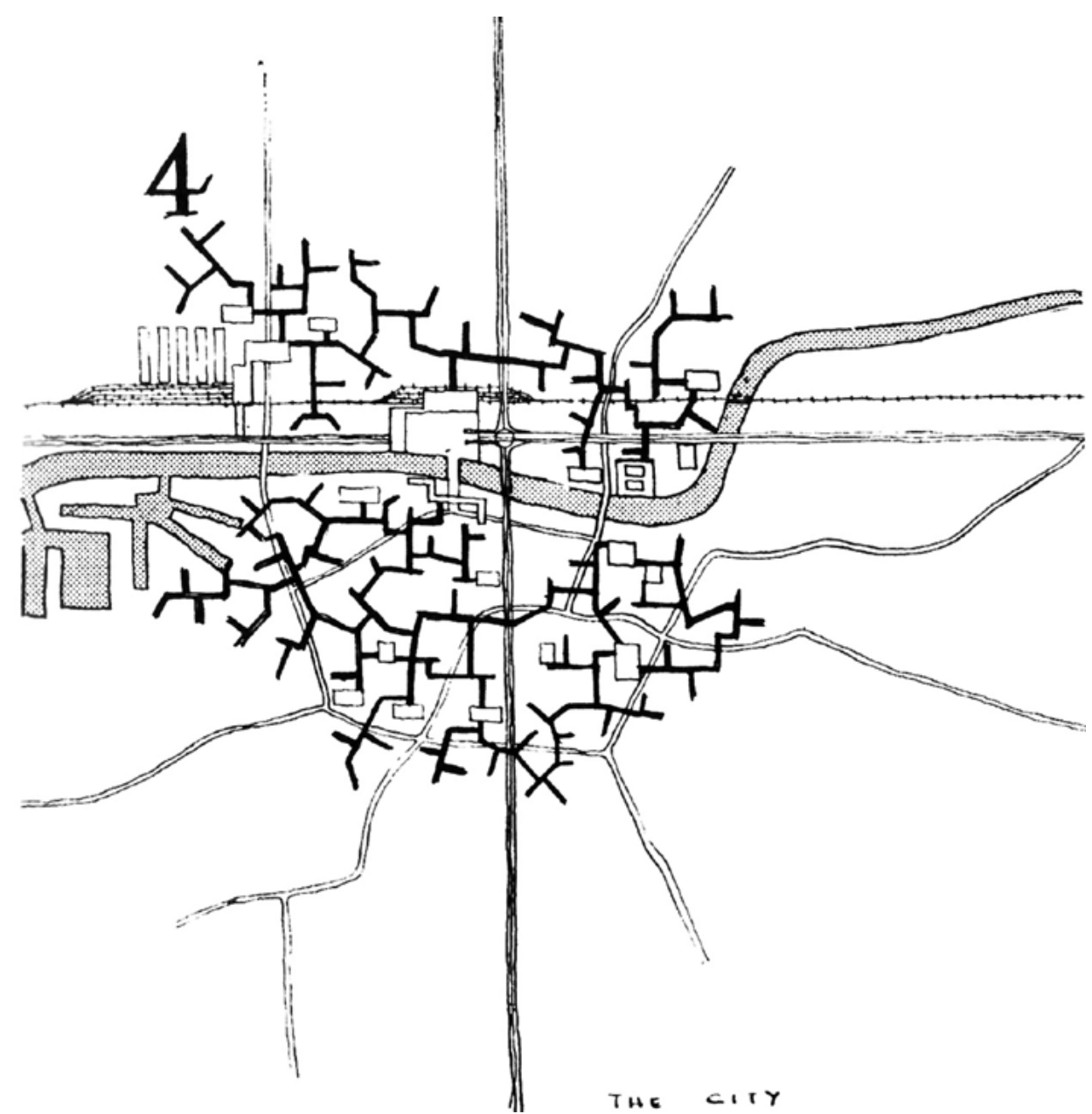

[6]

Ya vemos cómo los Smithson, conscientes de la importancia que está tomando la sociología, quieren utilizarla en el contexto arquitectónico. Y, desde luego, la calle no solo es el símbolo sino la primera forma de la arquitectura que defienden.

Otro dato que reafirma esta lectura se encuentra en uno de los fotomontajes que los Smithson realizan para la propuesta de concurso de Golden Lane (1953).

El lugar donde se desarrolla la escena no es otro que la calle exterior. Esta es una calle en altura. Las referencias a Le Corbusier son evidentes. Sin embargo, la gran evolución que plantea esta propuesta reside en que esta calle está volcada al exterior, comunicada visualmente con la ciudad y con otros tramos de la misma calle, y articula activamente distintos niveles del edificio -no es solo un espacio en cubierta-. Y, lo más importante, pasan cosas. De hecho, Marylin Monroe y Joe Dimaggio disfrutan del momento en esta "calle-en-el-aire", la cual sustituye a la "calle-corredor" del arquitecto francés.

Así pues, podemos decir que este fotomontaje de Peter Smithson termina por convertir el edificio en una serie de calles elevadas en donde estas acaban convirtiéndose en lugares de expresión individual y colectiva. Es decir, acaban convirtiéndose en espacios públicos que, en su red de conexiones múltiples, llegan a construir la totalidad de la ciudad.

Los Smithson, así pues, empiezan observando en detalle y amando la calle "tal cual" viene dada a través de las fotografías de Nigel Henderson, la convierten en un símbolo con su Scale of Association, la transforman en arquitectura en su propuesta de concurso para Golden Lane y la acaban figurando en una ciudad en su dibujo The City.

Sin embargo, los Smithson también estarian utilizando el icono "calle" para otros fines. En el CIAM VIII, celebrado en Hoddesdon en 1951, la Piazza italiana se convertiria en el emblema favorito de la nueva sociedad civil de posguerra y en el icono del "Core of the City" -corazón de la ciudad-, lema oficial del congreso, el cual pretendía responder a las críticas del excesivo funcionalismo de las cuatro funciones de la Carta de Atenas y de su "Centro Cívico" -limitado exclusivamente a una agrupación de edificios administrativos- mediante la exploración del con-
[6] Alison y Peter Smithson, The City (La Ciudad), 1952. Fuente: SMITHSON, Alison SMITHSON, Peter. Urban structuring: studies of Alison \& Peter Smithson. New York: Studio Vista, 1967, p. 20.

[7] Alison and Peter Smithson, Planta Típica de "Calles-Cubierta", concurso para Golden Lane, 1952. Fuente: SMITHSON, Alison; SMITHSON, Peter. The charged void, Urbanism. New York: Monacelli Press, 2004 p. 92 .

[8] Peter Smithson, ideograma originador de la calle-ramita como una unidad del distrito, 1952. Fuente: SMITHSON, Alison; SMITHSON, Peter. The charged void, Urbanism. New York: Monacelli Press, 2004, p. 84.

[9] Peter Smithson, "Cluster" de callesramita que llega a convertirse en distrito: una red de "calles-en-el-aire", 1952. Fuente: SMITHSON, Alison; SMITHSON, Peter. The charged void, Urbanism. New York: Monacelli Press, 2004, p. 84. 
15 Esto no significa que los Smithson negaran la importancia del concepto de "core" como el lugar de identificación simbólica de la "comunidad" a escala general de toda la ciudad, sino que, hábilmente, prefieren tomar otros temas no tratados por el resto como estructuradores de su discurso.

16 Los Smithson también criticarian los "Nine Points for a New Monumentality" de Sert, Giedion y Leger.

17 SMITHSON, Alison; SMITHSON, Peter. Urban structuring: studies of Alison \& Peter Smithson. New York: Studio Vista, 1967, p. 25

${ }^{18}$ El estructuralismo supuso un intencionado desplazamiento de los objetos hacia las relaciones entre ellos, un desplazamiento de la función a la estructura que permitiría comparaciones estructurales.

19 Esto es precisamente de lo que trata la Fenomenología.

20 "Doing without harmony and proportion" fue lo que dijo Peter Smithson en la inauguración de Parallel of Life and Art en 1953.

cepto de "urban core" -corazón de la ciudad-. En este contexto, los participantes en el CIAM VIII parecieron acordar la elección de la "Piazza" italiana como figuración máxima de todos los valores que representaban las distintas lecturas e interpretaciones del significado de "Core".

La sociología moderna del momento tenía su propio icono, la calle. Precisamente, la misma calle redescubierta a través de la cámara de Nigel Henderson y por los cientos de estudios sociológicos que se producían a pie de calle, día tras día, en el entorno de Bethnal Green, tan familiar para los Smithson, quienes, en un movimiento estratégico perfectamente planeado, sustituyen en su discurso la "Piazza" italiana, como figuración del "Core", por la calle de Bethnal Green. Con esto, los Smithson estarían haciendo otra sustitución ${ }^{15}$, casi más importante, la de "Monumentalidad" 16 por "Cotidianeidad".

\section{La construcción de la imagen de calle}

\section{- De la ortogonalidad controlada al libre albedrío}

"Una geometría más compleja que la "división parcelaria racional" responde a la necesidad de un entorno activo y creativo socialmente" ${ }^{17}$.

Alison y Peter Smithson eran muy conscientes de que la trama ortogonal y el sistema de información por capas eran dos de las estrategias habituales de representación del urbanismo hasta la fecha. Por esta razón, cualquier mensaje nuevo -en este caso, el de crear una ciudad a modo de entorno activo y creativo- necesitaría de una nueva imagen para poder calar más profundamente en el imaginario colectivo.

Una vez estaba claro que la calle sería el icono para construir la nueva imagen de ciudad, ¿qué forma específica adoptaría esta? Recordemos que la radicalidad de la ciudad planteada por los Smithson en The City hace desaparecer el plano del suelo casi totalmente a favor de una ciudad dibujada exclusivamente a partir de recorridos peatonales que nada tienen que ver con el planeamiento al uso. La calle en The City es una calle súper-conectada que parece trazar un recorrido arbitrario. Arbitrariedad en la elección del recorrido y conexión son, precisamente, las cualidades de su nuevo urbanismo, aquel que quiere construir un entorno humano multiescalar, activo y creativo.

La aparición del pensamiento relacional no es algo exclusivo de los Smithson. Esta idea de rechazar la composición a favor de la forma de las relaciones, es algo que algunos autores vinculan a la influencia de la recién aparecida ciencia de la cibernética. Otros, directamente, la vinculan con el pensamiento estructuralista ${ }^{18}$. No obstante, los Smithson fueron los primeros que desplazaron el centro de interés habitual por el objeto arquitectónico hacia las relaciones que este establece con su entorno humano ${ }^{19}$. En su caso, ya hemos visto que su entorno es cuádruple -casa, calle, distrito y ciudad- y que las relaciones que establece con él son de asociación -activa y creativa- entre personas. Así, la calle sería la primera figuración de la nueva arquitectura. Y la calle, conectada "arbitrariamente" rompiendo cualquier referencia a la ortogonalidad, acabaría construyendo la imagen de la nueva ciudad. De hecho, como Peter Smithson pronunciaría: "Haciendo sin harmonía ni proporción" 20.

La imagen de la figura 11 [11], de Eduardo Paolozzi, fue utilizada por los Smithson para la contraportada de su libro Urban Structuring. Si observamos esta pintura en detalle, como otra del mismo autor incluida en el interior de este libro, reconoceremos determinados fragmentos de la misma en el último dibujo de la serie y realizado por Alison Smithson. [12]

$[7$

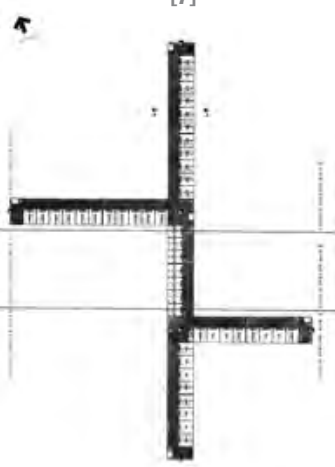

[8]

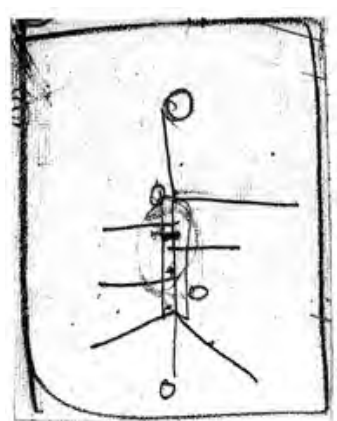

[9]

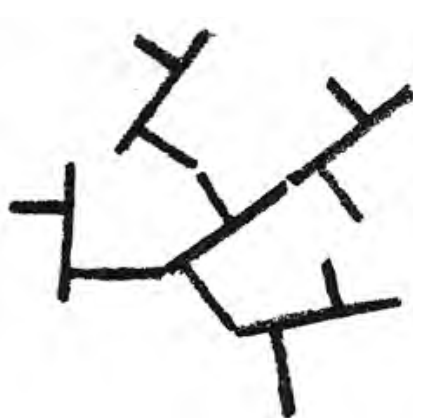


En Cluster City Alison Smithson nos muestra ahora una calle que cambia de espesor, que genera formas más complejas en determinados lugares, que se transforma en plaza...

"Uno especula... en nuestro trabajo, en parte cierto, que la "vida de las calles" -desde Nigel y Judith Henderson-, y las formas sueltas y enlazadas con las que comenzamos a formular estas ideas de la calle otra vez, en parte cierto, venían de los gráficos de Eduardo Paolozzi en aquel periodo" ${ }^{21}$.

\section{El uso del dibujo como construcción del deseo: concepto vs objeto}

"La intención de estos estudios era mostrar en términos de forma construida que una nueva aproximación al urbanismo era posible. En otras palabras, era presentar una imagen: una nueva estética era postulada así como un nuevo tipo de vida" ${ }^{22}$.

Los Smithson sabían que un nuevo sistema de referencias formales, como el de Paolozzi, les era necesario para poder construir una imagen suficientemente potente y novedosa para transmitir sus ideas. Hemos visto cómo la secuencia de dibujos previos y posteriores a Golden Lane sustituyen el concepto de asentamiento por el de asociación ${ }^{23}$, el urbanismo de la división -las cuatro funciones de la Carta de Atenas- por el urbanismo de la conexión, la Piazza italiana por la calle, el entorno pueblo/barrio/ciudad/gran ciudad/metrópolis por casa/calle/distrito/ciudad. Además, cogen la calle en altura de sus antecesores y la abren al exterior, la repiten en distintos pisos y la súper-conectan.

Por otro lado, podemos afirmar que eran conscientes de la necesidad de utilizar el dibujo de arquitectura no como herramienta de representación de un objeto, sino como herramienta de proposición de una idea. Es aquí donde los Smithson producen un salto cualitativo. Pensemos en el impacto que sus dibujos producirian en un contexto en el que cualquier idea se transmitía a partir de la construcción de un objeto arquitectónico o, en su defecto, de su representación. Los Smithson, de hecho, se refieren a esta circunstancia:

"Es importante darse cuenta que los términos utilizados -casa, calle, etc.- no deben ser tomados como realidad sino como una idea y es nuestra tarea la de encontrar nuevos equivalentes para estas formas de asociación para nuestra sociedad no-demostrativa" ${ }^{24}$.

Más relevante es, incluso, el hecho de que Alison y Peter Smithson se refiriesen a estos dibujos como ideogramas.

"Ideogramas por los que también reconocemos que, tanto en su orden como en su naturaleza, las formas a inventar necesitan ser conectivas" ${ }^{25}$.

Pero, sin duda, el hecho más evidente del nuevo uso del dibujo como construcción de una idea y no de un objeto serán las diferentes y múltiples interpretaciones de este dibujo que harán las generaciones posteriores. Un objeto arquitectónico es difícilmente indeterminado o no entendible. Es simplemente lo que es. Sin embargo, el grado de abstracción y contundencia al que los Smithson llegaron con Diagram of Appreciated Unit permitió iniciar una lectura
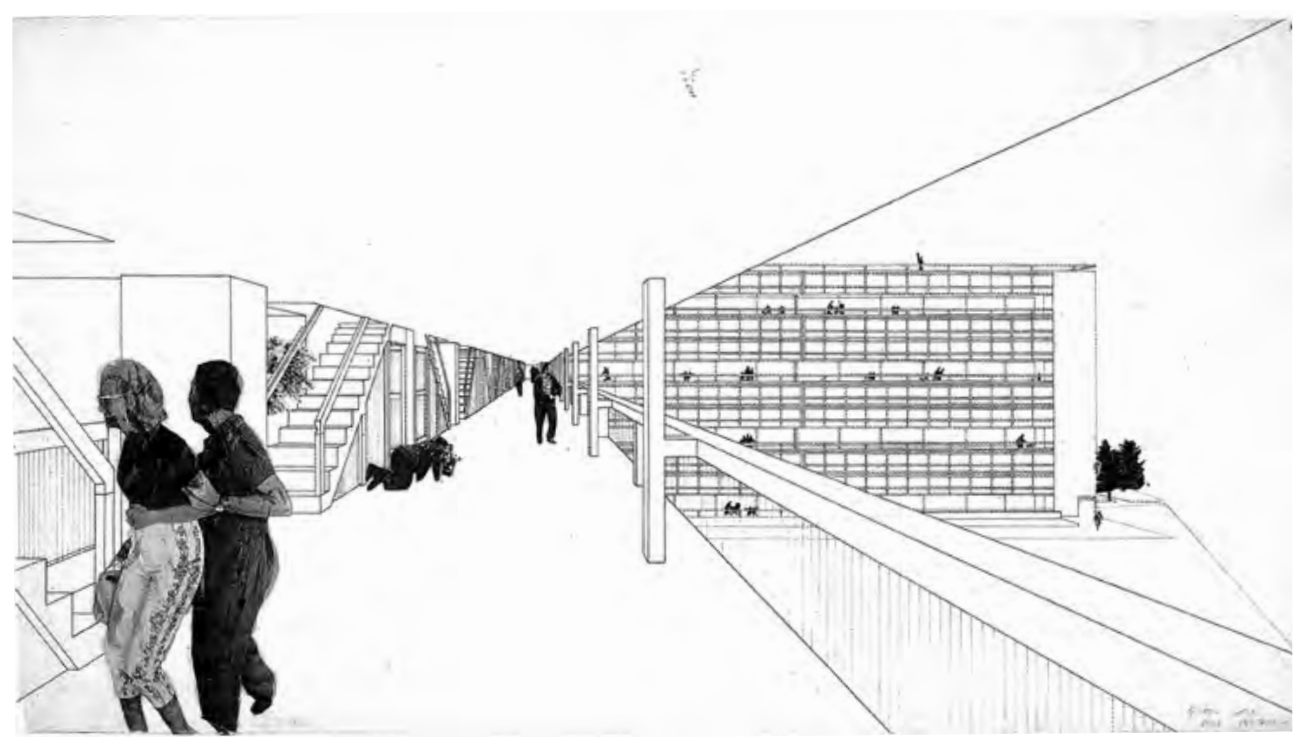

${ }^{21}$ WALSH, Victoria. Nigel Henderson : parallel of life and art. London: Thames \& Hudson, 2001, p. 151.

22 SMITHSON, Alison; SMITHSON, Peter. Urban structuring: studies of Alison \& Peter Smithson. New York: Studio Vista, 1967, p. 34

23 Ibid, p. 6.

24 Ibid, p. 21.

25 Ibíd, p. 130.

${ }^{26}$ SMITHSON, Peter. "Reflexions on Kenzo Tange's Tokyo Bay Plan". Architectural Design, octubre 1964.

27 GARCÍA GONZÁLEZ, Carlos. Atlas de Exodus. Director: Juan Herreros Guerra. E.T.S. Arquitectura (UPM), 2014.

${ }^{28}$ Collage, fotografías, tinta sobre papel y papiers collés. Ocho paneles, cada uno de $260 \mathrm{~cm} \times 55 \mathrm{~cm}$ enmarcados. Museo Nacional de Arte Moderno, París.
[10] Peter Smithson, fotomontaje de la propuesta para el concurso de Golden Lane, 1953. Fuente: SMITHSON, Alison: SMITHSON, Peter. The charged void, Urbanism. New York: Monacelli Press, 2004 p. 87.

[11] Eduardo Paolozzi, collage, 1951. Fuente: SMITHSON, Alison; SMITHSON, Peter. Urban structuring: studies of Alison \& Peter Smithson. New York: Studio Vista, 1967, p. 2.

[12] Alison Smithson, Cluster City 1952-53: Diagrammatic plan of a small city (Ciudad "Cluster" 1952-53: Planta diagramática de una ciudad pequeña), 1952-53. Fuente: SMITHSON, Alison; SMITHSON, Peter. Urban structuring: studies of Alison \& Peter Smithson. New York: Studio Vista, 1967, p. 26 .

[13] Comparativa elementos gráficos entre collage de Eduardo Paolozzi y Cluster City. Fuente: elaboración propia. 


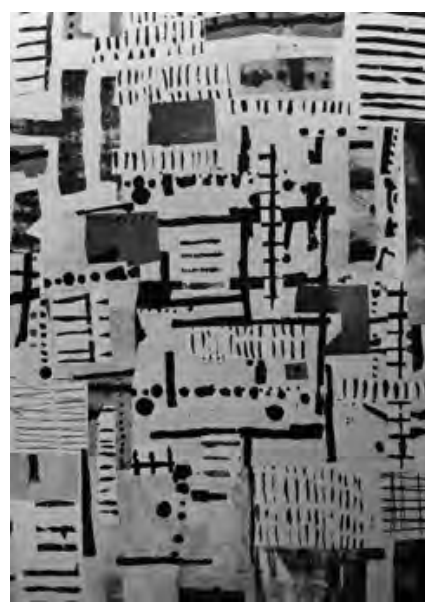

[11]

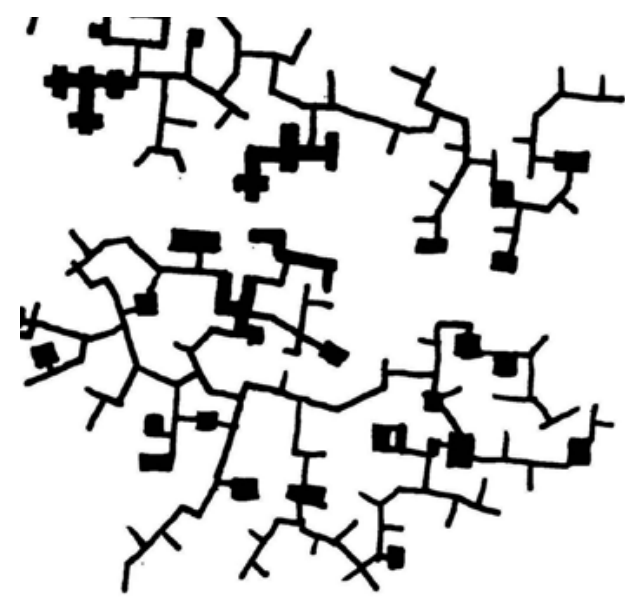

[12]

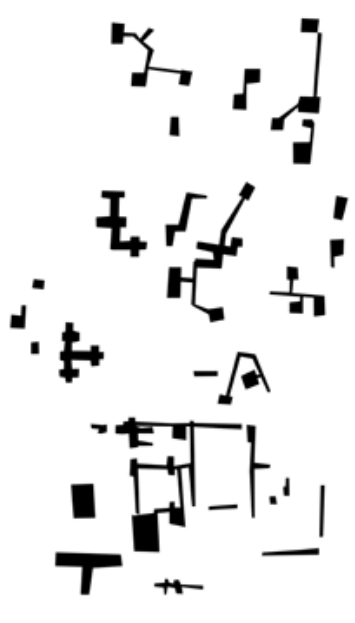

[13]

completamente nueva de nuestra ciudad y nuestro entorno y, sobre todo, del papel que la arquitectura debía jugar en su definición.

La toma de conciencia de la potencia de este nuevo uso del dibujo, probablemente, fue la que les llevó a radicalizar, más aún si cabe, el mensaje en el último de esta serie [13]. Vemos cómo la complejidad de formas se ha visto reducida de nuevo a la simplicidad de un solo trazado, un solo ancho y tipo de calle. Por otro lado, la ciudad existente sigue desaparecida. Los Smithson nos presentan un dibujo sin escala, sin localización, sin límites, extendido ad infinitum.

\section{Arquitectura como construcción de lo social. Lugar de "asociaciones"}

La influencia de la sociología en las prácticas arquitectónicas contemporáneas, así como la predominancia en algunas narrativas actuales de palabras como ecología, inclusividad, agonismo, cotidianeidad, arbitrariedad, indeterminación, relacional, lo ordinario... encuentran, de esta manera, gran parte de sus claves en el trabajo de los primeros años de los Smithson. De hecho, a través del trabajo de investigación de destacados autores -como Mark Wigley, Reyner Banham, Charles Jeckns, Kenneth Frampton, Barry Curtis, Manfredo Tafuri, Simon Sadler, Dominique Rouillard o William Menking- podemos establecer una continuación inmediata de sus planteamientos a través del trabajo de los llamados "Radicales" de los años 60 y 70. Los proyectos de "megaestructuras" -esta palabra sería utilizada por primera vez por Peter Smithson para referirse despectivamente al proyecto de la Bahía de Tokio de Kenzo Tange ${ }^{\mathbf{2 6}}$ - de personajes como Yona Friedman, Constant Nieuwenhuys o los Metabolistas japoneses comienzan a formalizar unos primeros esquemas de las ciudades relacionales de los Smithson, las cuales acabarán siendo desmanteladas -y reducidas a redes tecnológicas ocultas- por generaciones más jóvenes de grupos de arquitectos como Archigram, Superstudio, Archizoom... y, curiosamente, tomando como epicentro de este movimiento la Architectural Association de Londres. Será en esta escuela, precisamente, donde todos estos personajes propiciarán, de alguna manera, la aparición de un nuevo posicionamiento arquitectónico encarnado en las figuras de Leon Krier, Zaha Hadid, Bernard Tshumi o Rem Koolhaas. Carlos García González sitúa a este último, en el momento de presentación de su Proyecto Fin de Carrera Exodus, or the voluntary prisoners of Architecture (1972), como el principal personaje de esta generación capaz de formalizar a una escala menor -una década después y a través de ejemplos construidos convertidos en referentes arquitectónicos contemporáneos- el pensamiento relacional y asociativo de los Smithson.

La Arquitectura debe construir lugares en los que las personas puedan encontrarse, celebrar la cotidianeidad de sus vidas de manera subjetiva y no predeterminada, y donde manifestarse individual y colectivamente; pero, sobre todo, con libertad: la Arquitectura debe tener un carácter emancipador. Esto es, precisamente, lo que Alison y Peter Smithson reclaman. Diagram of Appreciated Unit es, así, la culminación de la ciudad entendida como tejido relacional. Sin embargo, no podemos olvidar que para los Smithson la ciudad es igual al barrio, a la calle y, por último, a la edificación. En otras palabras, los Smithson nos hablan de arquitectura y urbanismo al mismo tiempo y de manera indisoluble. Diagram of Appreciated Unit no dibuja ningún objeto sino relaciones, relaciones de asociación entre distintos entornos, entre personas. Los Smithson, al reducir la arquitectura a la calle, están reclamándole un papel más activo en la construcción de lo social a la vez que cierta condición pública, ya que esta comparte los mismos intereses con la sociedad que representa. Diagram of Appreciated Unit es, en definitiva, el comienzo de una gran historia todavía no concluida. 
04 | La Arquitectura como construcción de lo SOCIAL en los dibujos de Alison y Peter Smithson: De la calle como figuración de lo relacional a la desaparición de la escala y los límites_Iván Capdevila Castellanos

Alison y Peter Smithson en su Diagram of Appreciated Unit (1954) presentan un dibujo sin escala, sin localización, sin limites. Esta es la culminación de su ciudad entendida como tejido relacional. No dibujan ningún objeto sino relaciones de asociación entre distintos entornos y personas. De hecho, este posicionamiento a favor de un espacio relacional abstracto frente al de la composición formal provocará constante fricción entre las sucesivas generaciones desde los años 50 y 70 hasta el momento actual.

En paralelo, los Smithson, al reducir la arquitectura a la calle, reclaman un papel más activo de esta en la construcción de lo social. La arquitectura debe construir los lugares en los que las personas puedan encontrarse, celebrar la cotidianeidad de sus vidas, en donde manifestarse individual y colectivamente.

Son, precisamente, esta incorporación de lo público y del pensamiento inclusivo a la arquitectura, por un parte, y del entendimiento de esta como máquina relacional y posibilitadora de situaciones, por otra, los dos aspectos que confieren a su trabajo máxima actualidad. Este artículo indaga en las claves que determinan la formulación de ambos postulados a través de su gran herramienta epistemológica: sus dibujos.

\section{Palabras clave}

Social, arquitectura relacional, arquitectura inclusiva, Smithsons, calle, dibujo, cotidianidad, ecología, arbitrariedad
04 | Architecture as construction of the SOCIAL in Alison and Peter Smithson's drawings: From the street as figuration of the relational to the disappearance of the scale and boundaries_Iván Capdevila Castellanos

Alison and Peter Smithson in their Diagram of Appreciated Unit (1954) present a drawing without scale, without location, without limits. This is the culmination of their city understood as a relational fabric. They do not draw any object but associational relationships between different environments and people. In fact, this positioning in favour of an abstract relational space as opposed to that of formal composition will generate a constant friction between generations from the 50 s and 70 s up to the present time.

In parallel, the Smithson, by reducing the architecture to the street, claim a more active role in the construction of the social. Architecture must build the places where people can meet, celebrate the ordinariness of their lives, where they perform individually and collectively.

This incorporation of the public and inclusive thinking into architecture, on the one hand, and the understanding of it as a relational machine and enabler of situations, on the other, are the two aspects that make the work of the Smithson highly relevant today. This article investigates the keys that determine the formulation of both postulates through their great epistemological tool: their drawings.

Keywords

Social, Relational Architecture, Inclusive Architecture, Smithsons, street, drawing, ordinariness, ecology, randomness

\section{4 | La Arquitectura como construcción de lo SOCIAL en los dibujos de Alison y Peter Smithson: De la calle como figuración de lo relacional a la desaparición de la escala y los límites_Iván Capdevila Castellanos}

GEDDES, Patrick; THOMSON, J. Arthur. Evolution. New York: H. Holt and Co, 1911.

HENDERSON, Nigel. Photographs of Bethnal Green 1949-1952. Notthingham: Midland Group, 1978.

MASSEY, Anne. The Independent Group: modernism and mass culture in Britain, 1945-59. Manchester, New York: Manchester University Press, 1995. PAOLOZZI, Eduardo. Eduardo Paolozzi: artificial horizons and eccentric ladders: works on paper 1946-1995. London: British Council, 1996. RISSELADA, Max. VAN DEN HEUVEL, Dirk (eds). Team 10: 1953-81, in search of a utopia of the present. Rotterdam: NAi, 2005. ROBBINS, David (ed). The Independent Group: postwar Britain and the aesthetics of plenty. Cambridge Mass.: MIT Press, 1990. SMITHSON, Alison; SMITHSON, Peter. Urban structuring: studies of Alison \& Peter Smithson. New York: Studio Vista, 1967. SMITHSON, Alison; SMITHSON, Peter. The charged void, Urbanism. New York: Monacelli Press, 2004. WALSH, Victoria. Nigel Henderson: parallel of life and art. London: Thames \& Hudson, 2001. 